\title{
TWENTY THREE GAUGE VITRECTOMY IN MANAGEMENT OF DROPPED NUCLEUS AFTER PHACOEMULSIFICATION
}

\author{
Abd Ellatif, E. ${ }^{1{ }^{(*)}}$, Abd El-badie, M. ${ }^{1}$, Lashein, M. ${ }^{2}$ \& Abd El Karim, A. ${ }^{1}$ \\ ${ }^{1,2}$ Ophthalmology dept., Faculty of Medicine, AL-Azhar Univ., Assuit, Egypt \\ ${ }^{3}$ Ophthalmology dept, Faculty of Medicine, AL-Azhar Univ. Cairo, Egypt \\ *E-mail: dr.essam_ophth@yahoo.com
}

\begin{abstract}
Purpose: To evaluate the use of 23 gauge pars plana vitrectomy in management of dropped nucleus after phacoemulsification by two different techniques according to dropped nucleus size and consistency. Patients and Methods: Thirty eyes of thirty patients with dropped nucleus during phacoemulsification divided into two groups; each group consists of fifteen eyes. Group $\boldsymbol{A}$ includes 15 eyes performed using transconjunctival sutureless 23 gauge pars plana vitrectomy system with the use of PFCL and full removal of all dropped nuclear fragment by the vitreous cutter .Group B includes 15 eyes performed using transconjunctival suture less 23 gauge pars plana vitrectomy system with the use of PFCL and removal of some dropped nuclear fragment by the vitreous cutter while the others were removed through a limbal incision or scleral frown. Results: In group $\boldsymbol{A}$ we found that the mean VA was improved from 0.3 preoperatively to 0.3, 0.7 and 0.7 at $1^{\text {st }}$ week, 3rdand 6 months postoperatively respectively. In group $B$ we found that the mean VA was improved from 0.2 preoperatively to $1^{\text {st }}$ week, $3 r d$ and 6 months postoperatively respectively. In group $\boldsymbol{A}$ we found that the mean IOP was changed from $16 \mathrm{mmHg}$ at the baseline to $19 \mathrm{mmHg}, 17 \mathrm{mmHg}$ and $16 \mathrm{mmHg}$ at ${ }^{\text {st }}$ week, 3rd and 6 months postoperatively respectively. In group $\mathbf{B}$ we found that the mean IOP was changed from $18 \mathrm{mmHg}$ at the baseline to $17 \mathrm{mmHg}, 18 \mathrm{mmHg}$ and $16 \mathrm{mmHg}$ at $1^{\text {st }}$ week, 3rd and 6 months postoperatively respectively. Conclusion: Twenty three gauge pars plana vitrectomy was successful in cases of dropped fragments or quadrants and being soft cataract, while if it was all of the nucleus we can minimize its size by the vitreous cutter, and removing the remaining parts through a limbal wound or scleral frown trying to preserve the sutureless procedure as possible.
\end{abstract}

Keywords: Pars plana vitrectomy, Dropped nucleus, Phacoemulsification

\section{Introduction}

Cataract is the major cause of blind ness, accounting for approximately half of the world's blindness [1]. Phacoemulsification has become the most preferred method during the past two centuries [2]. However, dislocation of lens material fragments into the vitreous cavity after zonular dehiscence or posterior capsule rupture is actually uncommon, it is a serious complication owing to its subsequent glaucoma, chronic uveitis, retinal detachment and chronic cystoids macular oedema [3]. 
In one research, the general incidence of nucleus dropped to the vitreous was $0.8 \%$. Dropped nucleus management is controversial with regard to indications of su rgery, timing, longterm outcomes, and $\mathrm{m}$ edical management scope [4]. Currently, conservative strategy can be used only in the situation of small fragments, where medical management of inflammation and glaucoma can allow time for the fragment to be absorbed. Vitrectomy deals with the removing of fragment(s), hastening visual recovery, managing secondary complications and dealing with the expectations of the patients. [5]. Twenty three gauge pars plana vitrectomy is a sutureless vitrectomy procedure and it's a variation of

\section{Patients and Methods}

This prospective study was performed in Ophthalmology Department of Al-Azhar University Hospital-Assiut in the period from January 2014 to August 2014. Patients were randomly divided into two groups: $\mathrm{A}$ and $\mathrm{B}$ and included 30 eyes of 30 patients

\subsection{Inclusion criteria}

Patients underwent phacoemulsification complicated with dropped lens fragment or nucleus within two weeks of

\subsection{Exclusion criteria}

Patients with resultant retinal detachment as a result of dropped nucleus, patients with glaucoma and ocular infections, patients who had hard brown cataract before phacoemulsification and patients with opacified cornea. All patients were undergone ophthalmological examination of the eye including ocular examination with slit lamp biomicroscopy, uncorrected visual acuity (UCVA), best corrected visual acuity (BCVA), intraocular pressure (IOP) measurements, detailed fundus examination and B scan ultrasonography. The studied eyes were divided into 2 groups: each group consists of fifteen eyes. Group A includes 15 eyes performed using transconjunctival sutureless 23 gauge pars plana the 25-guage TSV system, was described firstly by Eckardt. Regardless of the instrumental measurement, the advantages of sutureless vitrectomy are comparable to those of sutureless cataract phacoemulsification including reduction of intraoperative time, patient discomfort, and postoperative inflammation. In addition, less surgical induced astigmatism and faster visual recovery were reported [6]. The aim of the work: is to evaluate the use of 23 gauge pars plana vitrectomy in management of dropped nucleus after phacoemulsification by two different techniques according to dropped nucleus size and consistency

with dropped nucleus during phacoemulsification. Approval of scientific committee of the Faculty of medicine, Al-Azhar university-Assiut, Egypt was obtained. A written informed consent was also obtained from each participant before the study.

presentation, age above 50 years and flat retina.

vitrectomy system with the use of PFCL (Per Flouro Carbon Liquid) and full removal of all dropped nuclear fragment using the vitreous cutter.Group B includes 15 eyes performed using transconjunctival sutureless 23 gauge pars plana vitrectomy system with the use of PFCL and removal of some dropped nuclear fragment using the vitreous cutter while the others were removed through a limbal incision or scleral frown. Pupillodilatation, local or general anesthesia and eye disinfection were carried out, insertion of Trocars then performing core vitrectomy, induction of PVD before injection of PFCL, instillation of a small PFCL bubble so that the macula is protected from the nucleus. Soft lens 
material can be removed first with the vitreous cutter, approximately 400 cuts/ min, fig (1). Aspiration of the lens fragments with the fluid needle and remove the fragments by the vitreous cutter, fig (2). This procedure is performed repeatedly until all the lens fragments are removed. Implantation of IOL: Three piece IOL implanted in the ciliary sulcus with or without optic capture (15 eyes). At the end, aspiration of the PFCL and removal of the Trocars. In group B injection of PFCL up to the sclerotomies, the nucleus is then pushed up to the level of the pupil. Then, injection of viscoelastic into the anterior chamber and luxate the dislocated nucleus into the anterior chamber with a 23-gauge membrane pic from the pars plana and a push pull from the paracentesis, injection of viscoelastic above the lens to protect the endothelium. The viscoelastic in the pupil can help to hold the lens fragments in the anterior chamber. Creation of an eight $\mathrm{mm}$ broad frown incision and extract the nucleus through the scleral frown using the lens scope. Implantation of IOL: hard IOL $(6.5 \mathrm{~mm})$ in the sulcus in cases with sufficient capsular rim (11 cases), iris claw IOL in cases with insufficient capsular rim (4 cases). At the end, aspiration of the PFCL and removal of the Trocars. Replacement of the conjunctiva over the scleral frown and closure by cauterization. Postoperatively patients were scheduled for follow up at $2^{\text {nd }}$ day to evaluate serious adverse effect like endophthalmitis and IOP elevation. Then at $1^{\text {st }}$ week, $3^{\text {rd }}$ week, 1 month, three months and six months postoperatively.

Figure (1) Shows the dropped nucleus is lifted and removed with the vitreous cutter.

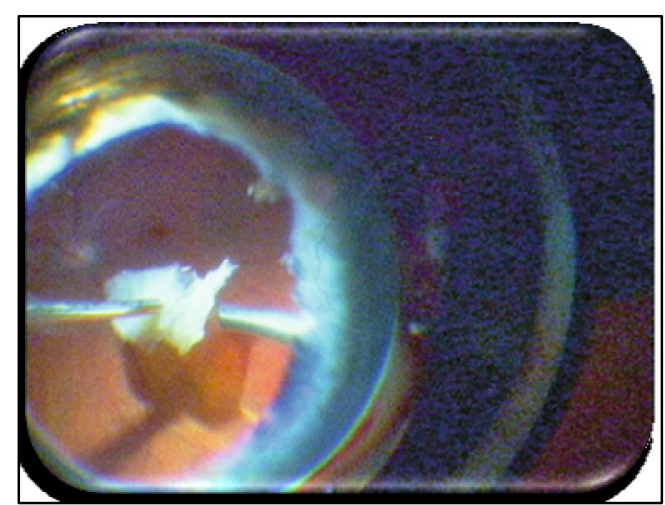

Figure (2) Shows the flute needle lifts a dislocated lens fragment from the retina to the centre of the vitreous cavity where it can be cut with the vitreous cutter without danger to the retina

\section{Statistical analysis}

Categorical data were delineated as number and percent $(\mathrm{N}, \%)$ while continuous data were existed as mean and standard deviation (Mean, SD). Chi-square test and t-test were used to compare between categorical variables and 
continuous variables respectively. Continuous data were analyzed for normal distribution using Kolmogorove Smirnov test and Q-Q Plots. A two-tailed $\mathrm{p}<0.05$

\section{Results}

After a mean follow-up of 6 months, final visual acuity ranged from $6 / 9$ to $6 / 18$ in 21 eyes $(70 \%)$ and $6 / 24$ to $6 / 36$ in 9 eyes $(30 \%)$. Visual rehabilitation was done with posterior chamber intraocular lens in 26 cases $(80 \%)$ and iris fixation IOL (Verisyse IOL) in 4 cases $(20 \%)$. Complications included: raised intraocular

\subsection{Demographic data}

All the patients included in the study are in the range of 50:60 years with mean age 55 years \& 4 months in group (A) and 53 years \& six months in group (B). Twelve cases in group (A) inc-

\subsection{Diabetes Mellitus}

Four cases in group (A) included in the study were diabetics, while eleven cases were non diabetic. In group (B) two cases

\subsection{Timing of the procedure}

In group (A): Ten cases underwent 23 PPV at the day zero due to the availability of the vitre-oretinal surgeon and the rest of cases were operated within the first ten days after phacoemulsification. In group (B): Nine cases were operated was considered statistically significant. All analyses were performed with the SPSS 20.0 software.

pressure in 6 patients (20\%), retinal detachment in 1 patient $(3.3 \%)$, reversible corneal oedema in 6 patients $(20 \%)$, cystoid macular oedema in 3 eyes (10\%). While corneal decompensation, post-operative hypotony and endophthalmitis were nil (0\%). RD occur in one case in group B, due to iatrogenic retinal breaks discovered postoperatively.

luded in the study were males and three cases were females, while in group (B); nine cases were males and six cases were females.

were diabetics, while thirteen cases were not diabetic.

at the day zero, while six cases were operated within the first ten days after phacoemulsification and nuclear drop due to the unavailability of vitre-oretinal surgeon, tab. (1).

Table (1)Timing of the procedure:

\begin{tabular}{|l|c|c|}
\hline & Group (A) & Group (B) \\
\hline Day zero & 10 & 9 \\
\hline Within 10 days & 5 & 6 \\
\hline
\end{tabular}

\subsection{Cause of nuclear drop}

In group (A): Thirteen cases were due to posterior capsular tear during phacoemulsification after sculpting and removal of two or three quadrants of cataract, while two cases were due to zonular dialysis due to pseudo exfoliation syndrome at the last piece. In group (B): Eleven cases were due

\subsection{IOL implanted}

In group (A): As all cases underwent total nuclear removal by the vitreous cutter, three piece IOL were implanted in the ciliary sulcus with or without optic to posterior capsular tear during phacoemulsification after sculpting, three cases were due to capsular block syndrome as a result of excessive hydrodissection on narrow capsulorhexis and one case was due to posterior capsular rupture due to hydrodissection for posterior polar cataract.

capture in the bag. In group (B): As all cases underwent nuclear remains removal though a scleral frown, hard IOL $(6.5 \mathrm{~mm}$ optic) were implanted in the ciliary 
sulcus in 11 cases and iris claw IOL in 4 cases due to absence of capsular rim

\subsection{Postoperative findings}

Visual acuity: The mean preoperative V/A was 0.3 in group (A) and 0.2 in group (B), tab. (2). After six months follow-up, the sufficient for support and stability of sulcus IOL.

mean postoperative V/A was 0.7 in group $\mathrm{A}$ and 0.6 in group $\mathrm{B}, \mathrm{P}$-value $=0.132$, fig. (3).

Table 2: The difference between the mean pre- and postoperative V/A in both groups.

\begin{tabular}{|l|c|c|}
\hline & Group (A) & Group (B) \\
\hline Mean preoperative V/A & $\mathbf{0 . 3}$ & $\mathbf{0 . 2}$ \\
\hline Mean postoperative V/A & $\mathbf{0 . 7}$ & $\mathbf{0 . 6}$ \\
\hline
\end{tabular}

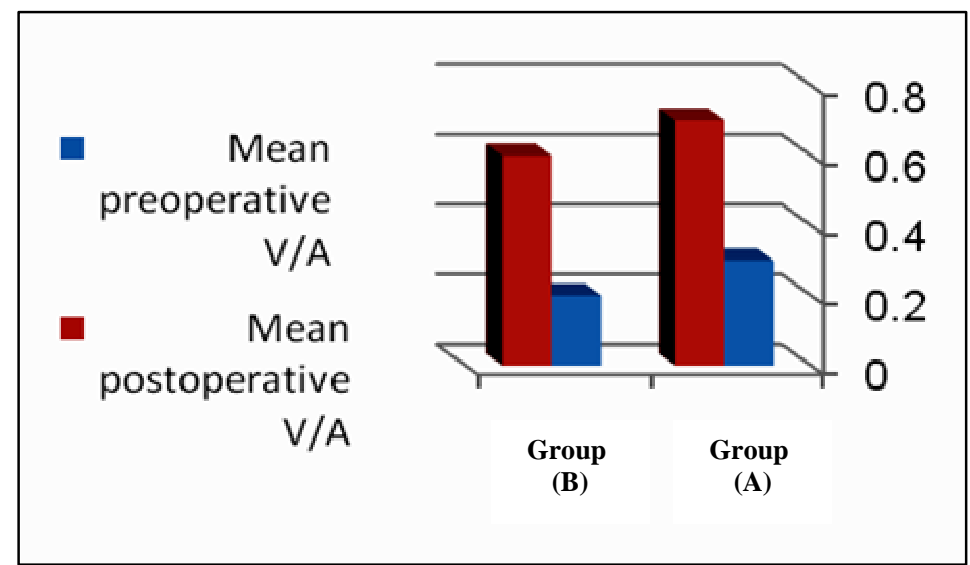

Figure (3) Shows the difference between the mean pre- and postoperative V/A in both groups

\subsection{IOP measurements}

Preoperative IOP Measurment: The mean preoperative IOP was $16 \mathrm{mmHg}$ in group (A) and $18 \mathrm{mmHg}$ in group (B). Postoperative IOP measurement: The mean first day IOP was $19 \mathrm{mmHg}$ in the group (A) vs $17 \mathrm{mmHg}$ in the group (B). Only one patient in the group (A) had a $26 \mathrm{mmHg}$ IOP recorded at the first day. In the group (B) however, two patients had IOP 26 and $28 \mathrm{mmHg}$ at day 1 and required a short course of oral acetazolamide and topical anti glaucoma treatment. NO patient in the group (A) had IOP recorded above $16 \mathrm{mmHg}$ with regular monitoring at the $1^{\text {st }}$ week, $3^{\text {rd }}$ week and $3^{\text {rd }}$ month post operatively. One patient in the group (B) had IOP recorded $26 \mathrm{mmHg}$ in the $1 \mathrm{st}$ week, which return to $24 \mathrm{~mm} \mathrm{Hg}$ at the $3^{\text {rd }}$ week and being $17 \mathrm{~mm} \mathrm{Hg}$ at the $3^{\text {rd }}$ month, controlled by topical antiglaucoma medications containing carbonic anhydrase enzyme and beta blocker agents e.g. Dorzolamide and Timolol combinations, fig (4).

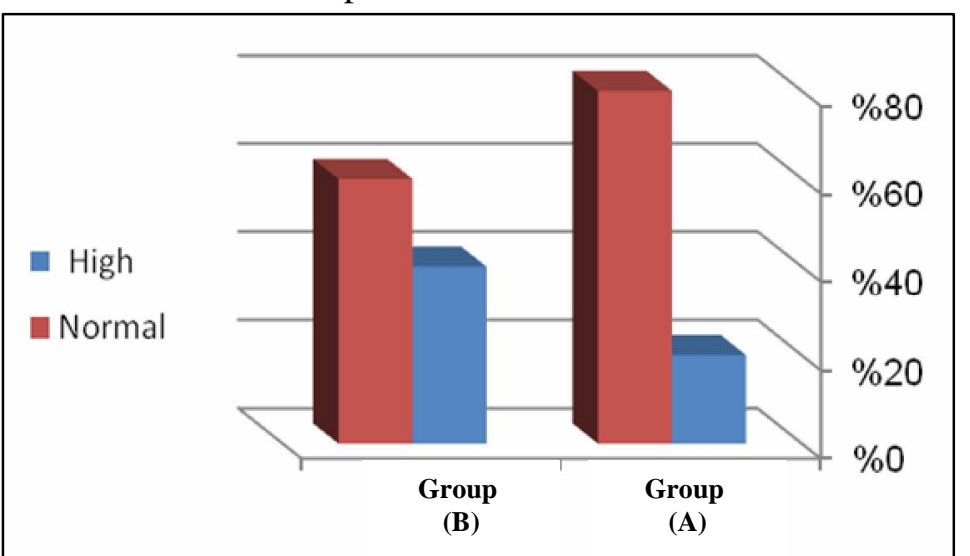

Fig.4: The difference between the mean pre- and postoperative IOP in both groups. 


\subsection{Anterior segment examination}

Slit-lamp examination in the $1^{\text {st }}$ postoperative day reveals 2 cases of reversible corneal edema in group- $A$ and 4 cases in group-B. Anterior chamber cells and flare were present in 2 cases in group-A and 4 cases in group-B. Iritis and pupillary mem- brane, fig (5) were present in only one casein group-A and 2 cases in group-B. Epithelial edema, fig (6) was present in 2 cases in group-A and 3 cases in group-B, tab. (3).

Table (3) Anterior segment examination.

\begin{tabular}{|l|c|c|}
\hline & Group (A) & Group (B) \\
\hline Corneal edema & 2 & 4 \\
\hline AC cells \& flare & 2 & 4 \\
\hline Iritis and membrane & 1 & 2 \\
\hline Epithelial edema & 2 & 3 \\
\hline
\end{tabular}

Figure (5) Shows postoperative uveitis and pupillary membrane.
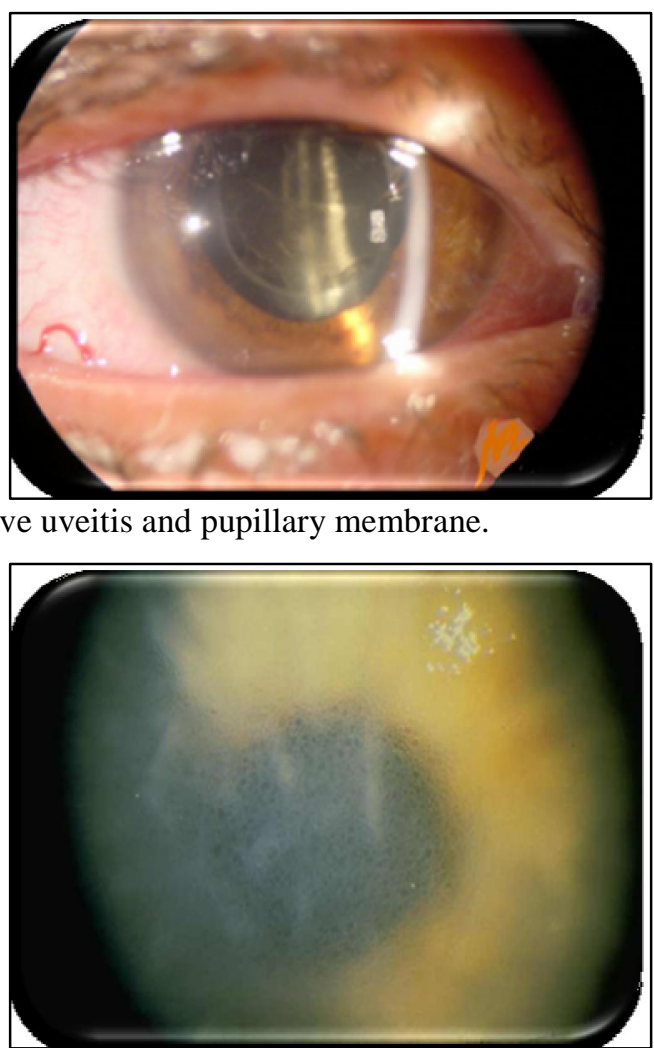

Figure (6) Shows postoperative epithelial edema due to high IOP

\section{9. latrogenic retinal break}

In group (A): no retinal breaks had seen at the end of surgery after 360 degree check of the peripheral retina. While In group (B): only one case showed iatrog-

\subsection{Post-operative hypotony}

All cases included in our study didn't show any post-operative hypotony with continuous measuring the IOP regularly

\subsection{Postoperative endophthalmitis}

All cases included in our study didn't show any post-operative endophthalmitis with regular examination at the $1^{\text {st }}$ day, enic break seen in the second postoperative day which is properly managed and encircled by laser and SO injection. The patient was high myope.

at the $1^{\text {st }}$ day, one week, three weeks, one month, three months and six months postoperatively.

one week, three weeks, one month, three months and six months postoperatively. 


\subsection{Cystoid macular edema}

Only one case in the group (B) showed post-operative cystoid macular edema confirmed by FFA and OCT which is then improved by medical treatment incl-

\section{Discussion}

\subsection{Visual acuity}

The mean preoperative V/A was 0.2. After follow-up of 6 months, $60 \%$ eyes had final visual acuity of (0.4) to (0.6). The mean postoperative V/A was 0.7 in group $\mathrm{A}$ and 0.6 in group $\mathrm{B}, \mathrm{P}$ value was 0.0132 with no significant statistical difference between the both groups. Tajunisah et al showed that the final visual acuity was 0.5 or better in $45.5 \%$ eyes. [7], while Verma et al reported that the final visual acuity was $>(0.3-0.4)$ in $75 \%$ of eyes [8]. The improvement of mean of visual acuity may be due to improvement of vitrectomy devices with less postoperative complications. Compared with 20-gauge PPV techniques, 23-gauge

\subsection{IOP measurements}

The mean preoperative IOP was 16 $\mathrm{mmHg}$, while the mean postoperative IOP was $19 \mathrm{mmHg}$. Only one patient had a first day $26 \mathrm{mmHg}$ IOP and received a short course of oral acetazolamide and topical anti glaucoma medications. No patients had postoperative hypotony (IOP

\section{3. latrogenic retinal break}

Only one case showed iatrogenic break seen in the second postoperative day which is properly managed and encircled by laser and SO injection. The patient was high myope. According to current beliefs, the vitreous clogging the

\subsection{Post-operative hypotony}

All cases included in our study didn't show any post-operative hypotony with continuous measuring the IOP regularly

\subsection{Post-operative endophthalmitis}

All cases included in our study didn't show any post-operative endophthalmitis with regular examination at the $1^{\text {st }}$ day, one week, three weeks, one month, three uding topical and systemic NSAIDs (e.g. Ketorline eye drops and Diclofenac $\mathrm{Na}$ tablets) and resolved after 6 weeks of continuous medications.

transconjunctival PPV: Eliminates the need for a conjunctival periotomy, reduce surgically induced sclerotomy trauma, corneal astigmatism, and mean operative times and reduce postoperative patient discomfort, intraocular inflammation and thereby allow for faster postoperative anatomical and visual recovery. Surgical induced astigmatism is minor in the early postoperative period after PPV with the 23-gauge TSV system. In comparison with the significant surgical induced astigmatism because of the scleral sutures in the early postoperative period after 20gauge PPV.

$>10 \mathrm{mmHg}$ ). Eckardt studied 41 eyes underwent PPV using the 23- gauge TSV system and reported that intraocular pressure on the first day postoperative never dropped for more than $12 \mathrm{mmHg}$, with no postoperative hypotony [6].

ports in TSV system could lead to increased rates of complications such as fibrovascular in-growth, recurrent vitreous hemorrhage and retinal breaks leading to detachment [9-12].

at the $1^{\text {st }}$ day, one week, three weeks, one month, three months and six months postoperatively.

months and six months postoperatively. Overall rates of postoperative endophthalmitis are quoted as $0.1 \%[13,14]$. 


\subsection{Cystoid macular edema}

Two cases in the showed postoperative cystoid macular edema confirmed by FFA and OCT which is then improved by medical treatment including topical and systemic NSAIDs (e.g. Ketorline eye drops and Diclofenac $\mathrm{Na}$ tablets) and resolved after 6 weeks of continuous medications. Postoperative cystoid macular oedema has been reported to occur in up to $27 \%$ in patients following pars plana vitrectomy for retained lens material. In this study, it was $10 \%$.

\section{Conclusion}

The management of dropped nucleus during cataract surgery using the 23 gauge cutter which could offer a better safety profile. This technique provides the total successful removal of all dropped nuclear fragments via a sutureless procedure allowing fast recovery and early visual rehabilitation. This procedure was successful in cases of dropped fragments or quadrants and being soft cataract.

\section{Ethical Considerations}

The study was approved by the scientific committee of the Faculty of medicine, Al-Azhar university- Assuit, Egypt. An informed written consent was taken from all the participants in the study.

\section{Acknowledgements}

We acknowledge all included participants, besides the clinical staff members of Ophthalmology Department, Al- Azhar University Hospital, Assuit, who helped the achievement of the study.

\section{References}

1. Ahmed, S., Shoaib, K., Fayyaz, M., Janjua, T. Positive impact of good visual outcome on the acceptance of cataract surgery in sub-Saharan African population. Pak J Ophthalmol. 2010; 26: 133-137.

2. Awan, A. Subjective assessment of pain level during phacoemulsification and extra capsular cataract extraction with intraocular lens implantation under topical anaesthesia. Pak J Ophthalmol. 2009; 25: 161-164.

3. Leaming, D. Practice styles and preferences of ASCRS members 1994 survey. J Cataract Refract Surg. 1995; 21: 378-385

4. Pande, M., Dabbs, T. Incidence of lens matter dislocation during phacoemulsification. J Cataract Refract Surg. 1996; 22: 737-742

5. Boscher, C., Lebuisson, D., Lean J., Nguyen-Khoa, J. Vitrectomy with endoscopy for management of retained lens fragments and/or posteriorly dislocated intraocular lens. Graefes Arch Clin Exp Ophthalmol. 1998; 236: 115-211.
6. Eckardt, C. Transconjunctival sutureless 23-gauge vitrectomy. Retina. 2005; 25 : 208-211.

7. Tajunisah, I., Reddy, S. Dropped nucleus following phacoemulsification cataract surgery. Med J Malaysia. 2007; 62: 364-367.

8. Verma, L., Gogoi, M., Tewari, H, Kumar, A., Talwar, D. Comparative study of vitrectomy for dropped nucleus with and without the use of perfluorocarbon liquid. Clinical, electrophysiological and visual field outcomes. Acta Ophthalmol Scand. 2001; 79: 354-358.

9. Yeh, P., Yang, C., Yang, C., Huang, J. Cryotherapy of the anterior retina and sclerotomy sites in diabetic vitrectomy to prevent recurrent vitreous hemorrhage: An ultrasound biomicroscopy study. Ophthalmology 2005; 112: 2095-2102.

10. Hajji, Z., Rouillot, J., Roth, P., Grange, J. Should associated intraoperative and/or postoperative photocoagulation be systematic during or after vitrectomy for proliferative diabetic vitreoretinopathy? J Fr Ophtalmol. 2003; 26: 47-53. 
11. West, J., Gregor, Z. Fibrovascular ingrowth and recurrent haemorrhage following diabetic vitrectomy. $\boldsymbol{B r} \boldsymbol{J}$ Ophthalmol. 2000; 84: 822- 825.

12. Sabti, K., Kapusta, M., Mansour. M., et al. Ultrasound biomicroscopy of sclerotomy sites: The effect of vitreous shaving around sclerotomy sites during pars plana vitrectomy. Retina. 2001; 21: 464-468.

13. Moore, J., Scott, I., Flynn, H., Smiddy, W., Murray, T., Kim, J., Vilar, N., Per- eira, M., Jorge, R. Retinal detachment in eyes undergoing pars plana vitrectomy for removal of retained lens fragments. Ophthalmology. 2003; 110: 709-713; discussion by TM Aaberg, Jr., 713-714

14. Mamalis, N. Complications of foldable intraocular lenses requiring explantation or secondary intervention, survey update. J Cataract Refract Surg. 2002; 28: 2193-2201. 